\title{
Diversity and characterization of lignocellulolytic fungi isolated from oil palm empty fruit bunch, and identification of influencing factors of natural composting process
}

\begin{abstract}
Oil palm empty fruit bunch (EFB) is the most significant waste generated from the agricultural industry in Malaysia. Composting is one of the potential approaches to utilize EFB. However, composting of EFB is a time-consuming process, thus impractical for industrial application. The composting process can be shortened by introducing competent fungi into an optimal EFB composting system. This study was conducted to isolate and identify competent fungi that can naturally compost EFB. Samplings were carried out at eight different time points over a 20 -weeks experimental period. The physical properties of EFB samples such as $\mathrm{pH}$, residual oil content, and moisture content were measured and the EFB composting process that was indicated by the contents of cellulose, hemicellulose, and lignin were assessed. The fungal growth, distribution, and lignocellulolytic enzyme activities were evaluated. The results indicated that the changes in physical properties of EFB were correlated to the fungal growth. The gradual reduction in moisture content and residual oil, and the increment in $\mathrm{pH}$ values in EFB samples throughout the experimental period resulted in reduced fungal growth and diversity. Such phenomenon delayed EFB composting process as revealed by the changes in EFB lignin, hemicellulose, and cellulose contents. The most dominant and resilient fungi (Lichtheimia ramosa and Neurospora crassa) survived up to 16 weeks and were capable of producing various lignocellulolytic enzymes. Further understanding of these factors that would contribute to effective EFB composting could be useful for future industrial applications.
\end{abstract}

Keyword: EFB; Physical properties; Lignocellulolytic enzyme; Lignin; Hemicellulose; Cellulose 
